\title{
Sexualidad e histerectomía: diferencias entre un grupo de mujeres con y sin ooforectomía
}

\author{
María Teresa Urrutia S. PhD 1a, Alejandra Araya G. PhD 1a, Oslando Padilla P.2b \\ ${ }^{1}$ Departamento de Salud de la Mujer, Escuela de Enfermería, Pontificia Universidad Católica de Chile. ${ }^{2}$ Departamento \\ de Salud Pública, Facultad de Medicina, Pontificia Universidad Católica de Chile.
}

a Enfermera-Matrona, ${ }^{b}$ Estadístico.

Proyecto financiado por la Dirección de Investigación de la Escuela de Enfermería de la Pontificia Universidad Católica de Chile. La Institución no tuvo influencia en el diseño del estudio; en la recolección, análisis o interpretación de los datos; en la preparación, revisión o aprobación de este manuscrito.

\section{RESUMEN}

Antecedentes: La ooforectomía bilateral, al momento de la histerectomía, es comprendida como una medida profiláctica para cáncer de ovario convirtiéndose en el órgano sano más frecuentemente extraído. La extracción profiláctica de ovarios alcanza una frecuencia del $40-55 \%$ de los casos de histerectomía. Se ha demostrado que las mujeres con histerectomía y ooforectomía muestran con mayor frecuencia deterioro de la función sexual que aquellas con sólo histerectomía. Objetivo: Comparar las características de la función sexual de un grupo de mujeres histerectomizadas, con y sin extracción de ovarios, a los 6 meses posteriores a la cirugía. Método: Estudio analítico, longitudinal, prospectivo en 67 mujeres: 35 histerectomizadas $(\mathrm{GH})$ y 32 histerectomizadas con ooforectomía (GH-O). Para la recolección de los datos se aplicó un cuestionario al tercer día posterior a la cirugía, y repetido a los 6 meses para determinar las características de la función sexual. Resultados: Después del control de variables basales, se produce una diferencia significativa entre el $\mathrm{GH}-\mathrm{O}$ y el GH en cuanto a prevalencia y frecuencia de dispareunia al sexto mes, frecuencia de orgasmo, frecuencia de relaciones sexuales y grado de excitación sexual. Conclusiones: La decisión de realizar una ooforectomía debe ser enfrentada con precaución en el caso de las mujeres con bajo riesgo de cáncer de ovario, considerando el riesgo individual de alteraciones sexuales, así como también, el riego inherente a la cirugía.

\section{PALABRAS CLAVE: Sexualidad, ooforectomía, histerectomía}

\section{SUMMARY}

Background: Bilateral oophorectomy, practiced at the time of hysterectomy, is understood as a prophylactic measurement for ovarian cancer, becoming the most frequently extracted healthy organ. Prophylactic oophorectomy has been reported at a frequency of $40-55 \%$ cases of hysterectomy. It has been demonstrated that women show a decrease of the sexual function after hysterectomy with bilateral oophorectomy more frequently than the women with hysterectomy only. Aim: To compare the characteristics of the sexuality in a group of women with and without oophorectomy, at the time of surgery and at 6 months postoperative. Method: Analytical, longitudinal and prospective study of 67 women, 35 with hysterectomy (GH) and 32 hysterectomized and oophorectomized (GH-O). Two surveys, at the time of surgery and six months later were carried out to determine the sexual function. Results: A significant difference was found between the $\mathrm{GH}-\mathrm{O}$ 
group and the GH group in prevalence and frequency of dispareunia, frequency of orgasm, frequency of intercourse, and sexual excitation. Conclusions: The decision for oophorectomy must be made with caution in the case of the women with low risk of ovarian cancer, considering the individual risk of sexual dysfunction as well as the risks inherent in the surgery.

\section{KEY WORDS: Sexuality, oophorectomy, hysterectomy}

\section{INTRODUCCIÓN}

La ooforectomía bilateral, practicada al momento de la histerectomía (HT), es comprendida rutinariamente como una medida profiláctica para cáncer de ovario en mujeres con riesgo genético desconocido (1-3) convirtiéndose en el órgano sano más frecuentemente extraído (1-2). Dicha decisión se ve además respaldada en la idea que los ovarios son considerados poco importantes para la mujer una vez finalizada su vida reproductiva (1). La extracción de ovarios profilácticamente comienza en la década de los años 30 (2), alcanzando en la actualidad el $40-55 \%$ de los casos de HT (4-6).

No hay duda en cuanto al beneficio que la ooforectomía profiláctica conlleva para la pequeña proporción de mujeres que tienen un riesgo genético de cáncer de ovario, sin embargo, para el grupo de mujeres sin ese riesgo, el balance entre ventajas y desventajas es materia de controversia (2,6-8).

La ooforectomía practicada en mujeres eugonadales produce hipogonadismo iatrogénico y consecuentemente causa una pérdida inmediata y significativa de niveles hormonales $(4,9-10)$ resultando en una disminución del $50 \%$ de los niveles de testosterona (11-17). Existe evidencia que los andrógenos son determinantes significativos de variables sexuales $(11,14-16,18-20)$, y se ha demostrado que las mujeres con HT y ooforectomía muestran una disminución de la función sexual más frecuentemente que las mujeres con HT sola $(3,6,13,20-23)$. Con respecto a la sexualidad de las mujeres histerectomizadas, la evidencia señala que no existen cambios en la frecuencia sexual (24), frecuencia de orgasmo y deseo sexual (25).

Esta comunicación forma parte de un estudio con publicaciones previas (25-27) en torno a la sexualidad de la mujer chilena histerectomizada. Se consideró hacer un análisis específico de este tópico, dada la carencia de reportes a nivel nacional. El objetivo del estudio es comparar algunas características de la función sexual de un grupo de mujeres histerectomizadas, con y sin extracción de ovarios, luego de un seguimiento a los 6 meses posteriores a la cirugía.

\section{MATERIAL Y MÉTODO}

Estudio analítico, longitudinal, prospectivo. Ingresaron al estudio 67 mujeres histerectomizadas, entre diciembre del 2002 y abril del 2003. Los criterios de inclusión fueron HT por patología ginecológica benigna, vida sexual activa y pareja estable en los últimos tres meses previos a la cirugía. Los criterios de exclusión fueron presencia de patología psiquiátrica diagnosticada o en tratamiento, menopausia prequirúrgica y usuaria de terapia de reemplazo hormonal $(\mathrm{TRH})$. Del total de mujeres ingresadas, 32 fueron histerectomizadas y oforectomizadas bilateralmente (GH-O) y 35 histerectomizadas (GH).

Para la recolección de los datos se realizó una entrevista estructurada al tercer día posterior a la cirugía en el hospital, la cual fue repetida a los 6 meses en el domicilio de la mujer. La entrevista fue realizada por Enfermeras-Matronas e internas de Enfermería-Obstetricia, quienes fueron entrenadas previamente. Ambas entrevistas fueron realizadas por la misma persona, con el objetivo de no incomodar y establecer una relación de confianza con la usuaria. Cabe señalar que las entrevistadoras no conocían el status gonadal de las mujeres entrevistadas.

Las variables estudiadas fueron: edad, nivel de escolaridad, tiempo de convivencia con la pareja, motivo de la HT, características de la cirugía y de la función sexual. Las variables de función sexual incluyen: presencia de orgasmo, dispareunia, e incontinencia urinaria durante la relación sexual; frecuencia de relaciones sexuales, de orgasmo, de dispareunia e incontinencia urinaria durante la relación sexual; grado de excitación, deseo sexual, satisfacción sexual y percepción de cambio en su función sexual producto de la HT. La frecuencia de relaciones sexuales fue medida en una semana, la frecuencia de dispareunia, orgasmo e incontinencia fue medida en un total de 10 relaciones sexuales, el grado de excitación, el deseo y la satisfacción sexual fueron medidas con una escala de Likert de 5 alternativas. Para la recolección de las características de la vida sexual se aplicó una adaptación del instrumento original "Percepción de calidad de comunicación de pareja y de calidad de vida sexual" 
(28), previa autorización de la autora, validado en una población de estudio proveniente del mismo Servicio de salud. La adaptación del instrumento consideró seleccionar las preguntas más representativas de las diferentes dimensiones medidas en el instrumento original y excluir las preguntas relacionadas al sistema de valores sexuales y antecedentes traumáticos. El alpha de Cronbach del instrumento adaptado fue de 0,86.

Los datos se analizaron con los programas estadísticos SPSS 17.0 y programa R. Los datos se resumen mediante medias, desviación estándar y frecuencias absolutas y relativas. Se realizaron comparaciones de dos grupos mediante las pruebas de: t Student, Kruskal-Wallis, Chi-cuadrado y asociación lineal por lineal. Los análisis multivariados incluyeron análisis de regresión logística, regresión binomial, regresión de Poisson y regresión ordinal. Se consideró significativo un valor $p<0,05$.

Esta investigación contó con la aprobación del Comité de Ética de la Escuela de Enfermería de la Pontificia Universidad Católica de Chile. Se solicitó firmar voluntariamente consentimiento informado a cada una de las mujeres del estudio.

\section{RESULTADOS}

Las características de las mujeres y de la cirugía se presentan en la Tabla I. Se observa una diferencia de edad entre ambos grupos, siendo significativamente menor en el GH que en el GH-O. La causa principal de HT en ambos grupos, fue la miomatosis uterina. De acuerdo al tipo de cirugía, la prevalencia de HT abdominal presentó diferencias significativas entre grupos, siendo mayor en el $\mathrm{GH}-\mathrm{O}$.

El inicio promedio de la actividad sexual fue a los $49,7 \pm 19,6$ días post-HT en el $\mathrm{GH}$ y $41,4 \pm 13,7$ días en el $\mathrm{GH}-\mathrm{O}$, sin diferencias significativas entre grupos $(p=0,083)$. El total de mujeres sin actividad sexual al sexto mes post-HT corresponden al GH-O (4/4). Las causas referidas fueron: temor de la pareja, miedo al dolor, problemas de comunicación con la pareja e incontinencia urinaria posquirúrgica.

Las características de función sexual de ambos grupos se presentan en las Tablas II y III. Todas las variables sexuales no muestran diferencias significativas entre los grupos en la medición basal, sin embargo, al sexto mes las diferencias se hacen significativas en frecuencia de dispareunia, frecuencia de orgasmo, grado de excitación y grado de deseo sexual.

La comparación de la vida sexual al sexto mes con la prequirúrgica se presenta en la Tabla IV. EI $\mathrm{GH}-\mathrm{O}$ tiene una percepción de empeoramiento de su vida sexual mayor que el $\mathrm{GH}$, mientras que el $\mathrm{GH}$ percibe en mayor grado un mejoramiento de su vida sexual que el GH-O.

Se realizaron análisis de regresión logística para modelar las prevalencias de dispareunia, incontinencia y orgasmo a los 6 meses controlando por los valores basales. A los análisis ingresaron las variables: edad; tiempo de convivencia; diagnóstico de mioma y prolapso; presencia de ooforectomía, de cirugía uro-ginecológica y de cirugía

Tabla I

CARACTERÍSTICAS DE LAS MUJERES EN ESTUDIO Y DE LA CIRUGÍA REALIZADA

\begin{tabular}{|c|c|c|c|}
\hline Variable & $\begin{array}{c}\mathrm{GH} \\
(\mathrm{n}=35)\end{array}$ & $\begin{array}{l}\mathrm{GH}-\mathrm{O} \\
(\mathrm{n}=32)\end{array}$ & Valor $\mathrm{p}$ \\
\hline Edad (años) & $42,80 \pm 3,99$ & $45,84 \pm 4,49$ & $<0,01$ \\
\hline Escolaridad (años) & $9,43 \pm 3,00$ & $8,41 \pm 2,92$ & NS \\
\hline Tiempo de convivencia (años) & $18,31 \pm 8,60$ & $20,97 \pm 9,89$ & NS \\
\hline $\begin{array}{l}\text { Diagnóstico de miomatosis como causa principal de la } \\
\text { histerectomía (\%) }\end{array}$ & 74,3 & 65,6 & NS \\
\hline $\begin{array}{l}\text { Diagnóstico de prolapso genital como causa principal } \\
\text { de la histerectomía (\%) }\end{array}$ & 22,9 & 6,3 & NS \\
\hline HT abdominal como vía de abordaje de la cirugía (\%) & 48,6 & 96,9 & $<0,01$ \\
\hline Presencia de cirugía del piso pélvico asociada a la HT (\%) & 20,0 & 6,3 & NS \\
\hline Presencia de cirugía uroginecológica asociada a la HT (\%) & 8,6 & 9,4 & NS \\
\hline
\end{tabular}

HT: Histerectomía. GH: Grupo con histerectomía. GH-O: Grupo con histerectomía y ooforectomía bilateral. NS: diferencia no significativa 
del piso pélvico, y las variables correspondientes en su medición basal. Los resultados se presentan en la Tabla V. La prevalencia de dispareunia al sexto mes resulta ser mayor en el GH-O con cirugía del piso pélvico aún cuando se controla por la dispareunia basal, variable que está directamente relacionada con la presencia de dispareunia al sex- to mes. Cabe destacar que el $\mathrm{GH}-\mathrm{O}$ presenta 8,4 veces mayor probabilidad de presentar un aumento en la prevalencia de dispareunia al sexto mes (EXP B: 8,42; IC95\%: 2,1-34,0), que el GH. En relación a la prevalencia de incontinencia y de orgasmo al sexto mes, ninguna de las variables ingresadas resultó significativa, no encontrándose una relación

Tabla II

CARACTERÍSTICAS DE LA SEXUALIDAD EN AMBOS GRUPOS DE ESTUDIO

\begin{tabular}{|c|c|c|c|c|}
\hline \multirow[b]{2}{*}{ Variable } & \multicolumn{4}{|c|}{ Grupo } \\
\hline & Tiempo & $\begin{array}{c}\mathrm{GH} \\
(n=35)\end{array}$ & $\begin{array}{l}\mathrm{GH}-\mathrm{O} \\
(\mathrm{n}=32)\end{array}$ & Valor $\mathrm{p}$ \\
\hline Frecuencia de relaciones sexuales (a) & $\begin{array}{c}\text { basal } \\
\text { al } 6^{\circ} \text { mes }\end{array}$ & $\begin{array}{l}1,96 \pm 1,71 \\
2,17 \pm 1,42\end{array}$ & $\begin{array}{l}2,40 \pm 1,83 \\
1,79 \pm 1,28\end{array}$ & $\begin{array}{l}0,225(c) \\
0,290(c)\end{array}$ \\
\hline Prevalencia de dispareunia & $\begin{array}{l}\text { basal } \\
\text { al } 6^{\circ} \text { mes }\end{array}$ & $\begin{array}{l}77,1 \% \\
22,9 \%\end{array}$ & $\begin{array}{l}59,4 \% \\
43,8 \%\end{array}$ & $\begin{array}{l}0,117(d) \\
0,069(d)\end{array}$ \\
\hline Frecuencia de dispareunia (b) & $\begin{array}{l}\text { basal } \\
\text { al } 6^{\circ} \text { mes }\end{array}$ & $\begin{array}{l}4,90 \pm 3,66 \\
0,91 \pm 2,43\end{array}$ & $\begin{array}{l}4,13 \pm 3,92 \\
2,31 \pm 3,50\end{array}$ & $\begin{array}{l}0,435(c) \\
0,049(c)\end{array}$ \\
\hline Prevalencia de incontinencia & $\begin{array}{c}\text { basal } \\
\text { al } 6^{\circ} \text { mes }\end{array}$ & $\begin{array}{c}14,3 \% \\
2,9 \%\end{array}$ & $\begin{array}{c}15,6 \% \\
9,4 \%\end{array}$ & $\begin{array}{l}0,878(d) \\
0,261(d)\end{array}$ \\
\hline Frecuencia de incontinencia (b) & $\begin{array}{l}\text { basal } \\
\text { al } 6^{\circ} \text { mes }\end{array}$ & $\begin{array}{l}0,57 \pm 1,89 \\
0,06 \pm 0,33\end{array}$ & $\begin{array}{l}0,61 \pm 1,57 \\
0,16 \pm 0,51\end{array}$ & $\begin{array}{l}0,839(c) \\
0,261(c)\end{array}$ \\
\hline Prevalencia de orgasmo & $\begin{array}{c}\text { basal } \\
\text { al } 6^{\circ} \text { mes }\end{array}$ & $\begin{array}{l}97,1 \% \\
94,3 \%\end{array}$ & $\begin{array}{l}96,9 \% \\
93,8 \%\end{array}$ & $\begin{array}{l}0,949(d) \\
0,926(d)\end{array}$ \\
\hline Frecuencia de orgasmo (b) & $\begin{array}{l}\text { basal } \\
\text { al } 6^{\circ} \mathrm{mes}\end{array}$ & $\begin{array}{l}6,10 \pm 2,73 \\
7,17 \pm 2,91\end{array}$ & $\begin{array}{l}5,60 \pm 3,03 \\
5,40 \pm 2,74\end{array}$ & $\begin{array}{l}0,470(c) \\
0,007(c)\end{array}$ \\
\hline
\end{tabular}

GH: Grupo con histerectomía. GH-O: Grupo con histerectomía y ooforectomía bilateral. (a) Frecuencia considerada en una semana. (b) Frecuencia considerada en un total de 10 relaciones sexuales. (c) Valor $p$ correspondiente a la prueba no paramétrica de Kruskal-Wallis. (d) Valor $\mathrm{p}$ correspondiente a la prueba Chi-cuadrado.

\section{Tabla III}

DISTRIBUCIÓN PORCENTUAL DEL GRADO DE EXCITACIÓN, DESEO Y GRADO DE SATISFACCIÓN SEXUAL EN AMBOS GRUPOS DE ESTUDIO

\begin{tabular}{|c|c|c|c|c|c|c|c|c|c|c|c|c|}
\hline & \multicolumn{4}{|c|}{ Grado de excitación } & \multicolumn{4}{|c|}{ Grado de deseo sexual } & \multicolumn{4}{|c|}{ Grado de satisfacción } \\
\hline & \multicolumn{2}{|c|}{ Basal } & \multicolumn{2}{|c|}{$6^{\circ}$ mes } & \multicolumn{2}{|c|}{ Basal } & \multicolumn{2}{|c|}{$6^{\circ}$ mes } & \multicolumn{2}{|c|}{ Basal } & \multicolumn{2}{|c|}{$6^{\circ}$ mes } \\
\hline & $\begin{array}{c}\mathrm{GH} \\
(\mathrm{n}=35)\end{array}$ & $\begin{array}{c}\mathrm{GH}-\mathrm{O} \\
(\mathrm{n}=32)\end{array}$ & $\begin{array}{c}\mathrm{GH} \\
(\mathrm{n}=35)\end{array}$ & $\begin{array}{c}\mathrm{GH}-\mathrm{O} \\
(\mathrm{n}=32)\end{array}$ & $\begin{array}{c}\text { GH } \\
(n=35)\end{array}$ & $\begin{array}{c}\mathrm{GH}-\mathrm{O} \\
(\mathrm{n}=32)\end{array}$ & $\begin{array}{c}\mathrm{GH} \\
(\mathrm{n}=35)\end{array}$ & $\begin{array}{l}\mathrm{GH}-\mathrm{O} \\
(\mathrm{n}=32)\end{array}$ & $\begin{array}{c}\mathrm{GH} \\
(\mathrm{n}=35)\end{array}$ & $\begin{array}{c}\mathrm{GH}-\mathrm{O} \\
(\mathrm{n}=32)\end{array}$ & $\begin{array}{c}\mathrm{GH} \\
(\mathrm{n}=35)\end{array}$ & $\begin{array}{l}\mathrm{GH}-\mathrm{O} \\
(\mathrm{n}=32)\end{array}$ \\
\hline Muy alto & $20,0 \%$ & $6,3 \%$ & $14,1 \%$ & $0 \%$ & $0,7 \%$ & $3,1 \%$ & $5,7 \%$ & $6,3 \%$ & $14,3 \%$ & $9,4 \%$ & $22,9 \%$ & $9,4 \%$ \\
\hline Alto & $42,9 \%$ & $62,5 \%$ & $57,1 \%$ & $50 \%$ & $40 \%$ & $21,9 \%$ & $54,3 \%$ & $21,9 \%$ & $57,1 \%$ & $56,3 \%$ & $60 \%$ & $59,4 \%$ \\
\hline Medio & $25,7 \%$ & $25 \%$ & $20 \%$ & $40,6 \%$ & $45,7 \%$ & $53,1 \%$ & $31,4 \%$ & $46,9 \%$ & $22,9 \%$ & $28,1 \%$ & $11,4 \%$ & $28,1 \%$ \\
\hline Bajo & $5,7 \%$ & $6,3 \%$ & $2,9 \%$ & $6,3 \%$ & $2,9 \%$ & $18,8 \%$ & $5,7 \%$ & $21,9 \%$ & $5,7 \%$ & $6,3 \%$ & $5,7 \%$ & $3,1 \%$ \\
\hline Muy bajo & $5,7 \%$ & $0 \%$ & $5,7 \%$ & $3,1 \%$ & $5,7 \%$ & $3,1 \%$ & $2,9 \%$ & $3,1 \%$ & $0 \%$ & $0 \%$ & $0 \%$ & $0 \%$ \\
\hline Valor $\mathrm{p}(\mathrm{a})$ & \multicolumn{2}{|c|}{0,859} & \multicolumn{2}{|c|}{0,038} & \multicolumn{2}{|c|}{0,055} & \multicolumn{2}{|c|}{0,013} & \multicolumn{2}{|c|}{0,514} & \multicolumn{2}{|c|}{0,097} \\
\hline
\end{tabular}

GH: Grupo con histerectomía. GH-O: Grupo con histerectomía y ooforectomía bilateral. (a) Valor p correspondiente a la prueba no paramétrica de Kruskal-Wallis. 
Tabla IV

DISTRIBUCIÓN PORCENTUAL DE LAS MUJERES SEGÚN COMPARACIÓN DE LA VIDA SEXUAL AL SEXTO MES CON LA QUE TENÍA ANTES DE LA CIRUGÍA

\begin{tabular}{lcccc}
\hline & \multicolumn{2}{c}{$\mathrm{GH}$} & \multicolumn{2}{c}{$\mathrm{GH}-\mathrm{O}$} \\
& $\mathrm{n}$ & $\%$ & $\mathrm{n}$ & $\%$ \\
\hline Mejor & 25 & 71,4 & 17 & 53,1 \\
Igual & 7 & 20,0 & 6 & 18,8 \\
Peor & 3 & 8,6 & 9 & 28,1 \\
TOTAL & 35 & 100 & 32 & 100 \\
\hline
\end{tabular}

GH: Grupo con histerectomía. GH-O: Grupo con histerectomía y ooforectomía bilateral. Valor $\mathrm{p}=0,048$ obtenido de la prueba Chi-cuadrado de asociación lineal por lineal

entre incontinencia y orgasmo al sexto mes con la extracción de los ovarios, después de controlar por las diferencias basales. Cabe señalar que en la prevalencia de orgasmo al sexto mes, el tiempo de convivencia presenta un valor $p$ límite $(p=0,055)$, señalando una tendencia hacia que a mayor tiempo de convivencia, la prevalencia de orgasmo es menor.
Según el análisis de regresión binomial (Tabla VI), la frecuencia de dispareunia al sexto mes es mayor en el $\mathrm{GH}-\mathrm{O}$, con diagnóstico de prolapso, sin cirugía uroginecológica, con menor grado de excitación al sexto mes y con mayor frecuencia de dispareunia basal. En particular, la frecuencia de dispareunia al sexto mes es mayor para $\mathrm{GH}-\mathrm{O}$, una vez que se ha controlado por la dispareunia basal y el grado de excitación. La frecuencia de incontinencia al sexto mes, es mayor en las mujeres con mayor frecuencia de incontinencia basal, después de controlar por dicha variable no se encuentran diferencias entre grupos. En relación a la frecuencia de orgasmo, esta es mayor en el GH con un mayor grado de excitación al sexto mes no dependiendo de la frecuencia de orgasmo basal.

En el análisis de la frecuencia de relaciones sexuales, la regresión de Poisson nos señala que el promedio de relaciones sexuales al sexto mes es mayor en el $\mathrm{GH}$, con mayor frecuencia basal, sin diagnóstico de mioma y prolapso, sin cirugía uro-ginecológica y con mayor grado de excitación al sexto mes. Cabe señalar que la edad de las mujeres muestra una tendencia que señala a menor edad de la mujer una mayor frecuencia de relaciones sexuales (Tabla VI).

Tabla V

REGRESIÓN LOGÍSTICA PARA VARIABLES PREVALENCIA DE DISPAREUNIA, INCONTINENCIA URINARIA Y ORGASMO AL SEXTO MES

\begin{tabular}{|c|c|c|c|c|c|}
\hline & \multicolumn{5}{|c|}{ Prevalencia de dispareunia al $6^{\circ}$ mes } \\
\hline & \multirow[b]{2}{*}{$\mathrm{B}$} & \multirow[b]{2}{*}{ Valor $\mathrm{p}$} & \multirow[b]{2}{*}{$\operatorname{Exp}(B)$} & \multicolumn{2}{|c|}{ IC $95 \%$ para $\operatorname{Exp}(B)$} \\
\hline & & & & Inferior & Superior \\
\hline Ooforectomía & 2,130 & 0,003 & 8,417 & 2,082 & 34,023 \\
\hline Cirugía piso pélvico & $-1,908$ & 0,035 & 0,148 & 0,025 & 0,873 \\
\hline Dispareunia basal & 3,614 & 0,002 & 37,127 & 3,749 & 367,706 \\
\hline \multirow[t]{4}{*}{ Constante } & $-3,104$ & 0,016 & 0,045 & & \\
\hline & \multicolumn{5}{|c|}{ Prevalencia de incontinencia al $6^{\circ}$ mes } \\
\hline & & & & \multicolumn{2}{|c|}{ IC $95 \%$ para $\operatorname{Exp}(B)$} \\
\hline & $\mathrm{B}$ & Valor $\mathrm{p}$ & $\operatorname{Exp}(B)$ & Inferior & Superior \\
\hline Ooforectomía & 1,294 & 0,287 & 3,647 & 0,337 & 39,442 \\
\hline Incontinencia basal & 1,956 & 0,073 & 7,073 & 0,831 & 60,173 \\
\hline \multirow[t]{4}{*}{ Constante } & $-4,114$ & 0,000 & 0,016 & & \\
\hline & \multicolumn{5}{|c|}{ Prevalencia de orgasmo al $6^{\circ}$ mes } \\
\hline & & & & \multicolumn{2}{|c|}{ IC $95 \%$ para $\operatorname{Exp}(B)$} \\
\hline & $\mathrm{B}$ & Valor $\mathrm{p}$ & $\operatorname{Exp}(B)$ & Inferior & Superior \\
\hline Años de convivencia & $-0,159$ & 0,055 & 0,853 & 0,724 & 0,1004 \\
\hline Constante & 6,653 & 0,006 & 775,096 & & \\
\hline
\end{tabular}


Tabla VI

REGRESIÓN BINOMIAL Y REGRESIÓN DE POISSON PARA VARIABLES FRECUENCIA DE DISPAREUNIA, INCONTINENCIA Y ORGASMO AL SEXTO MES

\begin{tabular}{|c|c|c|c|c|}
\hline & \multicolumn{4}{|c|}{ Frecuencia de dispareunia al $6^{\circ}$ mes (a) } \\
\hline & Estimación & Error Estándar & Valor Z & Valor $p$ \\
\hline Intercepto & $-8,4540$ & 2,1486 & $-3,935$ & $<0,001$ \\
\hline Ooforectomía & 1,3687 & 0,2644 & 5,176 & $<0,001$ \\
\hline Diagnóstico de prolapso & $-0,9459$ & 0,3259 & $-2,874$ & $<0,001$ \\
\hline Cirugía uroginecológica & 2,6471 & 1,0244 & 2,584 & 0,009 \\
\hline Excitación al $6^{\circ}$ mes & 0,7605 & 0,1276 & 5,957 & $<0,001$ \\
\hline \multirow[t]{2}{*}{ Dispareunia basal } & 0,1007 & 0,0337 & 2,987 & 0,003 \\
\hline & \multicolumn{4}{|c|}{ Frecuencia de incontinencia al $6^{\circ}$ mes (a) } \\
\hline Intercepto & $-5,5244$ & 0,8004 & $-6,902$ & $<0,001$ \\
\hline Ooforectomía & 1,1139 & 0,8593 & 1,296 & 0,1949 \\
\hline \multirow[t]{2}{*}{ Incontinencia Basal } & 0,2547 & 0,1277 & 1,995 & 0,0466 \\
\hline & \multicolumn{4}{|c|}{ Frecuencia de orgasmo al $6^{\circ}$ mes (a) } \\
\hline Intercepto & 2,8151 & 0,3965 & 7,100 & $<0,001$ \\
\hline Ooforectomía & $-0,5742$ & 0,1771 & $-3,242$ & 0,001 \\
\hline Diagnóstico de mioma & 0,3525 & 0,1994 & 1,768 & 0,077 \\
\hline \multirow[t]{2}{*}{ Excitación al $6^{\circ}$ mes } & $-0,9689$ & 0,1150 & $-8,423$ & $<0,001$ \\
\hline & \multicolumn{4}{|c|}{ Frecuencia de relaciones sexuales al $6^{\circ}$ mes $(b)$} \\
\hline Intercepto & $6,36 e+00$ & $1,372 e-01$ & 46,345 & $<0,001$ \\
\hline Ooforectomía & $-1,255 e-01$ & $2,213 e-02$ & $-5,673$ & $<0,001$ \\
\hline Edad & $-4,110 e-03$ & $2,255 e-03$ & $-1,822$ & 0,06 \\
\hline Frecuencia basal & $1,870 \mathrm{e}-03$ & $4,258 \mathrm{e}-05$ & 43,917 & $<0,001$ \\
\hline Diagnóstico de mioma & $5,850 \mathrm{e}-02$ & $2,301 e-02$ & 2,543 & 0,01 \\
\hline Diagnóstico de prolapso & $-1,796 e-01$ & $2,548 \mathrm{e}-02$ & $-7,048$ & $<0,001$ \\
\hline Cirugía uroginecológica & $2,358 \mathrm{e}-01$ & $3,090 e-02$ & $-7,633$ & $<0,001$ \\
\hline Excitación al $6^{\circ}$ mes & $2,597 e-01$ & $1,251 \mathrm{e}-02$ & $-20,752$ & $<0,001$ \\
\hline
\end{tabular}

(a) Regresión binomial. (b) Regresión de Poisson.

Tabla VII

REGRESIÓN ORDINAL PARA VARIABLES GRADO DE EXCITACIÓN, DESEO Y SATISFACCIÓN SEXUAL AL SEXTO MES

\begin{tabular}{|c|c|c|c|c|}
\hline & \multicolumn{4}{|c|}{ Excitación al Sexto Mes } \\
\hline & \multirow[b]{2}{*}{ Estimación } & \multirow[b]{2}{*}{ Valor $p$} & \multicolumn{2}{|c|}{ IC 95\% } \\
\hline & & & Inferior & Superior \\
\hline Excitación basal & 1,144 & $<0,001$ & 0,541 & 1,747 \\
\hline \multirow[t]{2}{*}{ Ooforectomía } & 1,383 & 0,009 & 0,342 & 2,424 \\
\hline & \multicolumn{4}{|c|}{ Deseo sexual al $6^{\circ}$ mes } \\
\hline Deseo sexual basal & 1,106 & $<0,001$ & 0,504 & 1,708 \\
\hline \multirow[t]{2}{*}{ Ooforectomía } & 0,931 & 0,057 & $-0,026$ & 1,887 \\
\hline & \multicolumn{4}{|c|}{ Satisfacción sexual } \\
\hline \multirow[t]{2}{*}{ Satisfacción sexual basal } & 1,135 & 0,001 & 0,437 & 1,832 \\
\hline & \multicolumn{4}{|c|}{ Comparación vida sexual } \\
\hline Ooforectomía & 0,831 & 0,096 & $-0,148$ & 1,810 \\
\hline
\end{tabular}


En cuanto al grado de excitación sexual (Tabla VII), la regresión ordinal señala que existe un mayor grado de excitación sexual al sexto mes cuando existe un mayor grado de excitación basal y cuando la mujer no tiene ooforectomía. En cuanto al grado de deseo sexual, este es mayor cuando existe un mayor grado de deseo sexual basal, si bien la ausencia de ooforectomía no es significativa, presenta un valor $\mathrm{p}$ límite que señala una cierta inclinación a señalar su efecto negativo. El mayor grado de satisfacción sexual al sexto mes sólo es explicado por el mayor grado de satisfacción basal. La comparación de la vida sexual al sexto mes, con la vida sexual basal, no presenta diferencias entre los grupos, a pesar que existe una tendencia a ser más favorable en el $\mathrm{GH}$.

\section{DISCUSIÓN}

La principal fortaleza del presente estudio, es que es el primer reporte de la sexualidad de mujeres chilenas histerectomizadas comparando ambos grupos de estudio. La principal debilidad es que es un estudio observacional, el que si bien controla estadísticamente aquellas variables influyentes, no tiene la fuerza de un estudio experimental.

Nuestro estudio coincide con Roovers y cols (24), que señalan un porcentaje menor de mujeres que no han iniciado actividad sexual en el $\mathrm{GH}-\mathrm{O}$, sin embargo, es diferente a lo reportado por otros investigadores (28-29). Este resultado podría considerarse como desfavorable para este grupo, ya que las mujeres y sus parejas son quienes están decidiendo respecto del reinicio de la actividad sexual, concepto denominado "alta subjetiva" (30).

La diferencia de edad entre ambos grupos de estudio ha sido reportada $(3,9,10,31)$ y es probable que sea un reflejo de los criterios utilizados al decidir la ooforectomía. Con respecto a la patología causal y características de la cirugía, los resultados coinciden también con otras publicaciones $(28,29,32,33,35)$.

La menor frecuencia de relaciones sexuales en el $\mathrm{GH}-\mathrm{O}$, ha sido reportada previamente $(3,21,23,29)$, y si bien depende de las características basales, esta variable puede ser un factor que influye negativamente en la percepción que la mujer tiene de su función sexual. El no tener actividad sexual puede transformarse en un parámetro que indica que la vida de pareja no ha retomado patrones prequirúrgicos, lo que genera expectación en la mujer.

La mayor prevalencia y frecuencia de dispareunia en el GH-O coincide con lo reportado por Bellerose y Binik (21) y Robson y cols (22), señalando el efecto negativo de la ooforectomía en este aspecto de la función sexual. El orgasmo ha sido establecido como una variable predictiva de la sexualidad post-HT (32), señalando que las mujeres con ooforectomía experimentan después de la cirugía una menor frecuencia de orgasmo posquirúrgica $(3,31)$, resultados coincidentes con este estudio observacional.

La diferencia en el deseo sexual entre ambos grupos ha sido reportada por otros investigadores $(6,21,23,31,36)$. Cabe señalar que la disminución del deseo sexual ha sido asociada a una menor frecuencia de orgasmo (31), menor frecuencia de relaciones sexuales (31) y menor grado de excitación $(6,21,23,31)$. Si bien en este estudio las diferencias no resultaron ser significativas entre grupos, después del control de las variables basales, la tendencia muestra cierta inclinación a mostrar un efecto negativo de la ooforectomía en esta variable, la que probablemente se haría presente al aumentar la muestra en estudio.

Resulta interesante señalar que a pesar de las diferencias en otras variables sexuales, las mujeres de ambos grupos coinciden en tener una mejor percepción de satisfacción sexual, variable que solo se ve influenciada por el grado de satisfacción sexual basal. Publicaciones internacionales han señalado un deterioro del deseo sexual en las mujeres sometidas a ooforectomía $(3,23)$, situación que no ocurrió en nuestro estudio. Este resultado puede ser explicado dado el largo tiempo que las mujeres presentan síntomas previo a la histerectomía, una reducción de la sintomatología prequirúrgica puede redundar en una mejor percepción de la satisfacción sexual de las mujeres en estudio.

La percepción negativa que las mujeres ooforectomizadas refieren en cuanto a su función sexual postcirugía puede estar explicada por los resultados a los cuales se ven enfrentadas (31). La ooforectomía ha sido reportada como una de las variables predictivas de mala calidad de función sexual a los 6 meses post-HT en mujeres chilenas eugonadales (25), y en este grupo en particular, podría explicar la tendencia existente a evaluar negativamente la vida sexual. Es necesario recordar que ninguna de las mujeres de este estudio era usuaria de $\mathrm{TRH}$ a los seis meses de seguimiento, sin embargo, aunque ellas cumplan con los criterios para ser usuarias de $\mathrm{TRH}$, el acceso a las terapias no siempre esta asegurado (7), por lo tanto, al momento de decidir la ooforectomía profiláctica, es otra variable que debiera ser considerada.

Es importante reconocer que el riesgo de cáncer de ovario ha sido sobre-enfatizado $(2,4,5)$. Al respecto Chiaffarino y cols (33), señalan que la HT 
con ooforectomía unilateral, tiene el mismo riesgo de desarrollar un cáncer de ovario que las mujeres con HT sin ooforectomía, y estas a su vez el mismo riesgo que las mujeres sin ninguna cirugía. Por otro lado, Parazzini (34) establece un efecto beneficioso de la histerectomía por si sola en la reducción del cáncer de ovario, sin embargo el aporte que hace la ooforectomía bilateral a la disminución del cáncer de ovario es esencialmente nulo.

Dentro de los beneficios de la conservación de ovarios se encuentra un menor riesgo de enfermedad cardiovascular $(4,5,7)$, fractura osteoporótica $(4,5,7,35)$, alteraciones sexuales $(5,14)$, alteraciones en la imagen corporal $(9,21)$ y síntomas menopáusicos $(4,5,14,37)$. En adición al conocido rol de los estrógenos, los andrógenos presentan también un rol importante en los diferentes órganos y sistemas $(3,4,9,11,13,14,16,19,31,35)$. Es importante recordar que después de la menopausia los ovarios continuarán produciendo andrógenos en cantidades significativas hasta los 80 años de edad $(4,5,14,17)$.

La ooforectomía profiláctica practicada junto a la HT ha sido definida como un problema de salud pública (1), y la decisión de realizarla debe ser enfrentada con precaución en el caso de las mujeres con bajo riesgo de cáncer de ovario $(1,4)$. Por lo anteriormente expuesto no se debería considerar sólo la edad de la mujer al momento de decidir dicha cirugía $(4,5)$, sino que también es necesario valorar el riesgo individual de la mujer para desarrollar un cáncer de ovario (4), así como los riesgos inherentes que la cirugía conlleva $(1,4,13,14,18,31,37,38)$ por la disminución hormonal que provoca sumado a la mayor expectativa de vida que las mujeres tienen actualmente.

Si frente a una valoración responsable y acuciosa de todos los factores, y en conjunto con la protagonista, se decide realizar la histerectomía con ooforectomía, se debe considerar la importancia de entregar información objetiva en relación a los efectos que la extracción de ovarios provoca $(4,9,12,14,18,20,23,37,39)$, especialmente sobre el dramático cambio desde el estado eugonadal a hipogonadal cuando se efectúa en mujeres que aún están menstruando, como también de las ventajas y desventajas de las alternativas hormonales recomendadas para el eventual reemplazo hormonal. De esta manera se asegura un mejor enfrentamiento de los cambios físicos y psicológicos a los cuales la mujer será expuesta $(29,40,41)$. Cabe señalar que actualmente en Chile no existe una norma que regule la información que debe ser entregada a la mujer antes de ser sometida a histerectomía y/o ooforectomía.
La extracción de los ovarios impacta la salud de la mujer en todas sus dimensiones y requiere, por lo tanto, que los profesionales de la salud estén preparados para enfrentar el cuidado a corto y largo plazo de este grupo de mujeres, dada la alta prevalencia de esta cirugía en nuestro país.

\section{CONCLUSIÓN}

La decisión de realizar una ooforectomía debe ser enfrentada con precaución en el caso de las mujeres con bajo riesgo de cáncer de ovario, considerando el riesgo individual de alteraciones sexuales, así como también, el riego inherente a la cirugía.

\section{BIBLIOGRAFÍA}

1. Clarke A, Chang YM, McPherson K. Removing organs "just in case"--is prophylactic removal of the ovaries a good thing? Epidemiol Community Health 2006;60(3):186-7.

2. Moscucci O, Clarke A. Prophylactic oophorectomy: a historical perspective. Epidemiol Community Health 2007;61(3):182-4.

3. Aziz A, Bergquist C, Brannstrom M, Nordholm L, Silfverstolpe G. Differences in aspects of personality and sexuality between perimenopausal women making different choices regarding prophylactic oophorectomy at elective hysterectomy. Acta Obstet Gynecol Scand Suppl 2005;84(9):854-9.

4. Parker WH, Broder MS, Liu Z, Shoupe D, Farquhar C, Berek JS. Ovarian conservation at the time of hysterectomy for benign disease. Clin Obstet Gynaecol 2007;50(2):354-61.

5. Parker WH, Shoupe D, Broder MS, Liu Z, Farquhar C, Berek JS. Elective oophorectomy in the gynecological patient: when is it desirable? Curr Opin Obstet Gynecol 2007;19(4):350-4.

6. McPherson K, Herbert A, Judge A, Clarke A, Bridgman $S$, Maresh $M$, et al. Psychosexual health 5 years after hysterectomy: population-based comparison with endometrial ablation for dysfunctional uterine bleeding. Health Expect 2005;8(3):234-43.

7. Charoenkwan K, Srisomboon J, Suprasert P, Phongnarisorn C, Siriaree S, Cheewakriangkrai C. Role of prophylactic oophorectomy at the time of hysterectomy in ovarian cancer prevention in Thailand. J Obstet Gynaecol Res 2004;30(1):20-3.

8. Orozco LJ, Salazar A, Clarke J, Tristan M. Hysterectomy versus hysterectomy plus oophorectomy for premenopausal women. Cochrane Database Syst Rev 2008(3):CD005638.

9. Teplin V, Vittinghoff E, Lin F, Learman LA, Richter HE, Kuppermann M. Oophorectomy in premenopausal women: health-related quality of life and sexual functioning. Obstet Gynecol 2007;109(2 Pt 1):347-54.

10. Aziz A, Bergquist C, Nordholm L, Moller A, Silfverstolpe G. Prophylactic oophorectomy at elective hysterectomy. Effects on psychological well-being at 1-year 
follow-up and its correlations to sexuality. Maturitas 2005;51(4):349-57.

11. Apperloo MJ, Van Der Stege JG, Hoek A, Weijmar Schultz WC. In the mood for sex: the value of androgens. J Sex Marital Ther 2003;29(2):87-102; discussion 77-9.

12. Shifren JL, Avis NE. Surgical menopause: effects on psychological well-being and sexuality. Menopause 2007;14(3 Pt 2):586-91.

13. Kalantaridou SN, Calis KA. Testosterone therapy in premenopausal women. Semin Reprod Med 2006;24(2):106-14.

14. Nappi RE, Wawra K, Schmitt S. Hypoactive sexual desire disorder in postmenopausal women. Gynecol Endocrinol 2006;22(6):318-23.

15. Braunstein GD, Sundwall DA, Katz M, Shifren JL, Buster JE, Simon JA, et al. Safety and efficacy of a testosterone patch for the treatment of hypoactive sexual desire disorder in surgically menopausal women: a randomized, placebo-controlled trial. Arch Intern Med 2005; 165(14):1582-9.

16. Cameron DR, Braunstein GD. Androgen replacement therapy in women. Fertil Steril 2004;82(2):273-89.

17. Laughlin GA, Barrett-Connor E, Kritz-Silverstein D, von Muhlen D. Hysterectomy, oophorectomy, and endogenous sex hormone levels in older women: the Rancho Bernardo Study. J Clin Endocrinol Metab 2000;85(2):645-51.

18. Elit L, Esplen MJ, Butler K, Narod S. Quality of life and psychosexual adjustment after prophylactic oophorectomy for a family history of ovarian cancer. Fam Cancer 2001;1(3-4):149-56.

19. Buster JE, Kingsberg SA, Aguirre O, Brown C, Breaux JG, Buch A, et al. Testosterone patch for low sexual desire in surgically menopausal women: a randomized trial. Obstet Gynecol 2005;105(5 Pt 1):944-52.

20. Shifren JL. Androgen deficiency in the oophorectomized woman. Fertil Steril 2002;77 Suppl 4:S60-2.

21. Bellerose SB, Binik YM. Body image and sexuality in oophorectomized women. Arch Sex Behav 1993;22(5):435-59.

22. Robson M, Hensley M, Barakat R, Brown C, Chi D, Poynor E, et al. Quality of life in women at risk for ovarian cancer who have undergone risk-reducing oophorectomy. Gynecol Oncol 2003;89(2):281-7.

23. Nathorst-Boos J, von Schoultz B. Psychological reactions and sexual life after hysterectomy with and without oophorectomy. Gynecol Obstet Invest 1992;34(2):97-101.

24. Roovers JP, van der Bom JG, van der Vaart $\mathrm{CH}$, Heintz AP. Hysterectomy and sexual wellbeing: prospective observational study of vaginal hysterectomy, subtotal abdominal hysterectomy, and total abdominal hysterectomy. BMJ 2003;327(7418):774-8.

25. Urrutia MT, Araya A, Rivera S, Viviani P, Villarroel L. Sexualidad en la mujer histerectomizada: modelo predictivo al sexto mes posterior a la cirugía. Rev Méd Chile 2007;135(3):317-25.

26. Urrutia MT, Araya A, Villarroel L, Viñales D. Caracterís- ticas y evolución de la sexualidad en mujeres histerectomizadas. Rev Chil Obstet Ginecol 2004;69(4):301-6.

27. Urrutia MT, Araya A. Sexualidad en mujeres histerectomizadas al tercer mes posterior a la cirugía. Rev Chil Obstet Ginecol 2005;70(3):160-5.

28. Rivera MS. Vida sexual de mujeres de estrato social medio bajo y bajo y su relación con la comunicación y conflictos de pareja, sistema de valores sexuales y antecedentes traumáticos sexuales [Tesis de Magister en salud Pública, Mención epidemiología]. Santiago, Chile: Universidad de Chile; 1986.

29. Williams RD, Clark AJ. A qualitative study of women's hysterectomy experience. J Womens Health Gend Based Med 2000;9 Suppl 2:S15-25.

30. Urrutia MT, Riquelme P, Araya A. Educación de mujeres histerectomizadas ¿Qué desean saber? Rev Chil Obstet Ginecol 2006;71(6):410-6.

31. Leiblum SR, Koochaki PE, Rodenberg CA, Barton IP, Rosen RC. Hypoactive sexual desire disorder in postmenopausal women: US results from the Women's International Study of Health and Sexuality (WISHeS). Menopause 2006;13(1):46-56.

32. Helström L, Lundberg PO, Sorbom D, Backstrom T. Sexuality after hysterectomy: a factor analysis of women's sexual lives before and after subtotal hysterectomy. Obstet Gynecol 1993;81(3):357-62.

33. Chiaffarino F, Parazzini F, Decarli A, Franceschi S, Talamini R, Montella M, et al. Hysterectomy with or without unilateral oophorectomy and risk of ovarian cancer. Obstet Gynecol 2005;97(2):318-22.

34. Parazzini F, Negri E, La Vecchia C,Luchini L, Mezzopane R. Hysterectomy, oophorectomy, and subsequent ovarian cancer risk. Obstet Gynecol 1993;81(3):363-6.

35. Leao LM, Duarte MP, Farias ML. [Female androgen insufficiency and potential risks of therapeutic replacement]. Arq Bras Endocrinol Metabol 2005;49(2):205-16.

36. Nappi RE, Lello S, Melis GB, Albani F, Polatti F, Genazzani AR. LEI (Lack of testosterone Impact) survey in a clinical sample with surgical menopause. Climacteric 2009;12(6):533-40.

37. Farquhar CM, Harvey SA, Yu Y, Sadler L, Stewart AW. A prospective study of 3 years of outcomes after hysterectomy with and without oophorectomy. Am J Obstet Gynecol 2006;194(3):711-7.

38. Rocca WA, Grossardt BR, de Andrade M, Malkasian GD, Melton LJ, 3rd. Survival patterns after oophorectomy in premenopausal women: a population-based cohort study. Lancet Oncol 2006;7(10):821-8.

39. Fry A, Busby-Earle C, Rush R, Cull A. Prophylactic oophorectomy versus screening: psychosocial outcomes in women at increased risk of ovarian cancer. Psychooncology 2001;10(3):231-41.

40. Liu HE, Ya SF. The immediate and short-term effects of an educational program for post radical hysterectomy self-care of the lower urinary tract. Chang Gung Med J 2001;24(7):440-5.

41. Lindberg CE, Nolan LB. Women's decision making regarding hysterectomy. J Obstet Gynecol Neonatal Nurs 2001;30(6):607-16. 